\title{
On the scattering problem in Ryckman's class of Jacobi matrices
}

\author{
Leonid Golinskii, Alexander Kheifets ${ }^{1}$ and Petro Yuditskii ${ }^{2}$
}

\begin{abstract}
We define and characterize the scattering data for a class of Jacobi matrices that was recently introduced by E. Ryckman. We prove the uniqueness and give a complete solution to the inverse scattering problem in this class.
\end{abstract}

Mathematics Subject Classification (2010). Primary 46L37; Secondary 57M99.

Keywords. Scattering data, Besov class, Szegő asymptotics, Jost solution, inverse scattering problem.

\section{Introduction}

In mid 70s Guseinov [8], [9] developed a scattering theory for infinite Jacobi matrices

$$
J=J\left(\left\{a_{n}\right\},\left\{b_{n}\right\}\right)=\left[\begin{array}{cccc}
b_{1} & a_{1} & 0 & \ldots \\
a_{1} & b_{2} & a_{2} & \ldots \\
0 & a_{2} & b_{3} & \ldots \\
\vdots & \vdots & \vdots & \ddots
\end{array}\right],
$$

$a_{n}>0, b_{n}=\bar{b}_{n}$, which can be viewed as a discrete version of the scattering theory for one-dimensional Schrödinger operator on the half-line by Marchenko-Faddeev. The basic assumption on $J$ is the finiteness of the first moment

$$
\sum_{n=1}^{\infty} n\left(\left|a_{n}-1\right|+\left|b_{n}\right|\right)<\infty .
$$

We say that a Jacobi matrix $J=J\left(\left\{a_{n}\right\},\left\{b_{n}\right\}\right)$ belongs to Guseinov's class $\mathcal{E}$ if its entries satisfy (2). Later Geronimo [4], [5] (see also [6]) solved the spectral problem for Jacobi matrices in more general "weighted" Guseinov's classes by using the inverse scattering technique. The main feature of his results is that the decay of the

\footnotetext{
${ }^{1}$ The work was partially supported by the University of Massachusetts Lowell Research and Scholarship Grant, project number: H50090000000010.

${ }^{2}$ The work was partially supported by the Austrian Science Found FWF, project number: P22025-N18.
} 
Jacobi parameters $\left\{a_{n}-1\right\},\left\{b_{n}\right\}$ manifests itself in the decay of the Fourier coefficients of the absolutely continuous part of the measure (after suitable modifications).

In the modern scattering theory of Jacobi operators (see, e.g., [3]) the various analogues of (2) (for much more complex backgrounds) appeared, and they seemed to be indispensable.

In 2007 Ryckman [13], [14], [15] came up with a new class of Jacobi matrices, for which he obtained a complete spectral description. To state his result we introduce some notations and definitions. Let us write

$$
\beta=\left\{\beta_{n}\right\} \in \ell_{s}^{2}, \quad s>0 \quad \text { if } \quad\|\beta\|_{\ell_{s}^{2}}^{2}=\sum_{n}|n|^{s}\left|\beta_{n}\right|^{2}<\infty .
$$

Definition 1.1. A Jacobi matrix $J=J\left(\left\{a_{n}\right\},\left\{b_{n}\right\}\right)$ belongs to Ryckman's class $\mathcal{R}$, or its spectral measure $\sigma(J) \in \mathcal{R}$, if the series $\sum_{n}\left(a_{n}-1\right)$ and $\sum_{n} b_{n}$ are conditionally summable, and $\xi=\left\{\xi_{n}\right\} \in \ell_{1}^{2}, \eta=\left\{\eta_{n}\right\} \in \ell_{1}^{2}$ with

$$
\xi_{n}=-\sum_{k=n+1}^{\infty} b_{k}, \quad \eta_{n}=-\sum_{k=n+1}^{\infty}\left(a_{k}-1\right) .
$$

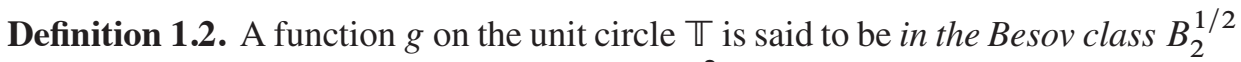
if the sequence of its Fourier coefficients is in $\ell_{1}^{2}(\mathbb{Z})$

$$
g(t)=\sum_{n \in \mathbb{Z}} g_{n} t^{n}, \quad \sum_{n \in \mathbb{Z}}|n|\left|g_{n}\right|^{2}<\infty .
$$

Let $f$ be a function on the interval $[-2,2]$. By $\hat{f}$ we will mean a unique function

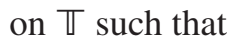

$$
\hat{f}(t)=f\left(t+\frac{1}{t}\right) .
$$

Note that $\hat{f}$ is a symmetric function, $\hat{f}(\bar{t})=\hat{f}(t)$, and conversely, every symmetric function on $\mathbb{T}$ is of the form $\hat{f}$. If

$$
h(t)=\sum_{n \in \mathbb{Z}} h_{n} t^{n},
$$

then the symmetry of $h$ implies that $h_{-n}=h_{n}$. If $h$ is in addition a real function, then $h_{-n}=h_{n}=\overline{h_{n}}$.

Definition 1.3. A function $f$ on the interval $[-2,2]$ is said to be in $B_{2}^{1 / 2}$ if the function $\hat{f}$, defined as in (4), is in $B_{2}^{1 / 2}$.

Theorem 1.4 (Ryckman). $J \in \mathcal{R}$ if and only if the spectral measure $\sigma(J)$ of $J$ has the following structure. 
- The absolutely continuous part is supported by [-2,2] and

$$
\begin{aligned}
\sigma_{a c}(d x) & =f(x, J) d x=\frac{\rho(x, J)}{2 \pi} \sqrt{4-x^{2}} d x, \\
\rho(x, J) & =\frac{\rho_{0}(x, J)}{(2-x)^{\gamma_{1}(J)}(2+x)^{\gamma_{2}(J)}},
\end{aligned}
$$

with $\gamma_{1}(J), \gamma_{2}(J)$ equal 0 or 1 , and $\log \rho_{0} \in B_{2}^{1 / 2}$.

- The singular part is

$$
\sigma_{s}(d x)=\sum_{k=1}^{N} \sigma_{k} \delta\left(\lambda_{k}\right)
$$

with $N=N(J)<\infty, \sigma_{k}(J)>0$, and $\lambda_{k}(J) \in \mathbb{R} \backslash[-2,2]$.

We single out the factor $\sqrt{4-x^{2}}$ to have simpler expressions in (18), (19) below. Note that $\mathscr{G} \subset \mathcal{R}$, and the inclusion is proper. Indeed,

$$
\begin{aligned}
\sum_{n=1}^{\infty} n\left|\xi_{n}\right|^{2} & =\sum_{n=1}^{\infty} n\left(\sum_{k=n+1}^{\infty} b_{k}\right)^{2} \leq \sum_{n=1}^{\infty} \sum_{k=n}^{\infty} \sum_{l=n}^{\infty} n\left|b_{k}\right|\left|b_{l}\right| \\
& \leq \sum_{n=1}^{\infty} \sum_{k=n}^{\infty} \sum_{l=n}^{\infty} l\left|b_{k}\right|\left|b_{l}\right| \leq \sum_{n=1}^{\infty} \sum_{k=n}^{\infty} \sum_{l=1}^{\infty} l\left|b_{k}\right|\left|b_{l}\right| \\
& =\sum_{k=1}^{\infty} \sum_{n=1}^{k} \sum_{l=1}^{\infty} l\left|b_{k}\right|\left|b_{l}\right|=\sum_{k=1}^{\infty} k \sum_{l=1}^{\infty} l\left|b_{k}\right|\left|b_{l}\right|=\left(\sum_{k=1}^{\infty} k\left|b_{k}\right|\right)^{2} .
\end{aligned}
$$

Similarly,

$$
\sum_{n=1}^{\infty} n\left|\eta_{n}\right|^{2} \leq\left(\sum_{k=1}^{\infty} k\left|a_{k}-1\right|\right)^{2}
$$

The inclusion $\mathcal{G} \subset \mathcal{R}$ is proper, since

$$
a_{n}=1+\frac{(-1)^{n}}{n \log (n+1)}, \quad b_{n}=\frac{(-1)^{n}}{n \log (n+1)}
$$

belongs to $\mathcal{R}$, but (2) fails ( $J$ is not even a trace class perturbation of the free Jacobi matrix $\left.J_{0}=J(\{1\},\{0\})\right)$.

Given $\sigma(J) \in \mathcal{R}$, we define (recall that the notation ^ was introduced in (4))

$$
D_{0}(z)=D_{0}(z, J)=\exp \left\{\frac{1}{2} \int_{\mathbb{T}} \frac{t+z}{t-z} \log \hat{\rho}_{0}(t, J) m(d t)\right\},
$$

$\left|D_{0}(t)\right|^{2}=\hat{\rho}_{0}(t, J)$ almost everywhere on $\mathbb{T}$. Since

$$
\hat{\rho}_{0}(t, J)=\hat{\rho}_{0}(\bar{t}, J),
$$


we have that

$$
D_{0}(\bar{z})=\overline{D_{0}(z)}
$$

We can write $D_{0}$ as

$$
D_{0}(z)=\exp \left\{\frac{u_{0}(z)+i v_{0}(z)}{2}\right\},
$$

where $u_{0}$ and $v_{0}$ are real valued harmonic functions. Again, due to (9), $u_{0}$ is symmetric, and $v_{0}$ is antisymmetric: $u_{0}(\bar{z})=u_{0}(z), v_{0}(\bar{z})=-v_{0}(z) . v_{0}$ is the harmonic conjugate to $u_{0}$,

$$
u_{0}(t)=\log \hat{\rho}_{0}(t, J)=\sum_{k \in \mathbb{Z}} u_{0, k} t^{k}, \quad v_{0}(t)=\tilde{u}_{0}(z)=\frac{1}{i} \sum_{k \in \mathbb{Z}}(\operatorname{sgn} k) u_{0, k} t^{k} .
$$

We will need the following known proposition (cf., e.g., [16, Proposition 6.1.5])

Proposition 1.5. Let $D_{0}$ be defined as in (8) with $\log \hat{\rho}_{0} \in B_{2}^{1 / 2}$. Then, for all $p<\infty,\left(\hat{\rho}_{0}\right)^{ \pm 1} \in L^{p}(\mathbb{T})$, so

$$
D_{0}^{ \pm 1} \in H^{p}, \quad \forall p<\infty
$$

We put next

$$
\begin{aligned}
D(z) & =D(z, J)=\exp \left\{\frac{1}{2} \int_{\mathbb{T}} \frac{t+z}{t-z} \log \hat{\rho}(t, J) m(d t)\right\} \\
& =\frac{D_{0}(z, J)}{(1-z)^{\gamma_{1}}(1+z)^{\gamma_{2}}},
\end{aligned}
$$

the last equality in (12) follows from the second equality in (5) with $x=t+1 / t$ and the identities

$$
2-t-\frac{1}{t}=|1-t|^{2}, \quad 2+t+\frac{1}{t}=|1+t|^{2}, \quad|t|=1 .
$$

Both $D_{0}$ and $D$ are related to the absolutely continuous part of the spectral measure. The discrete part is completely determined by the set of eigenvalues $\left\{\lambda_{k}\right\}$, or equivalently, by the set

$$
Z(J)=\left\{z_{k}(J): \lambda_{k}=z_{k}(J)+\frac{1}{z_{k}(J)}, k=1,2, \ldots, N\right\},
$$

$z_{k}(J) \in(-1,1) \backslash\{0\}$, and by the set of masses $\left\{\sigma_{k}(J)\right\}_{k=1}^{N}$ in (6).

Definition 1.6. Given $J \in \mathcal{R}$, under the scattering data for $J$ we mean the following collection $\left\{\gamma_{1}(J), \gamma_{2}(J) ; Z(J) ; \mu_{1}(J), \ldots, \mu_{N}(J) ; s(t, J)\right\}$ :

(1) a pair $\left(\gamma_{1}(J), \gamma_{2}(J)\right)$ from (5); 
(2) the set $Z(J)$ from (13), or equivalently, a finite Blaschke product

$$
\begin{gathered}
B(z, J)=\prod_{k=1}^{N} \frac{z_{k}(J)}{\left|z_{k}(J)\right|} \frac{z-z_{k}(J)}{1-z_{k}(J) z}, \\
B(\bar{t}, J)=\overline{B(t, J)}=\frac{1}{B(t, J)}, \quad t \in \mathbb{T} ;
\end{gathered}
$$

(3) $N=N(J)$ positive numbers

$$
\mu_{k}(J)=\frac{\sigma_{k}(J)}{\left|1-z_{k}^{-2}(J)\right|^{2}}\left|\frac{B^{\prime}\left(z_{k}, J\right)}{D\left(z_{k}, J\right)}\right|^{2}>0, \quad k=1,2, \ldots, N ;
$$

(4) the scattering function

$$
s(t, J)=\frac{\varphi_{0}(t, J)}{\varphi_{0}(\bar{t}, J)},
$$

where $\varphi_{0}$ is the Jost function for $J$ (see Section 2).

Compared to [4] and [5], we move backward, from spectral to scattering. The goal of the present note is to obtain a complete characterization of the scattering data in Ryckman's class, and so demonstrate that the scattering theory goes beyond Guseinov's class (2). We analyze the scattering data and prove the uniqueness theorem in Section 2. We solve the inverse scattering problem in Section 3.

\section{Scattering data}

The basic three-term recurrence relation for a Jacobi matrix $J\left(\left\{a_{n}\right\},\left\{b_{n}\right\}\right)$

$$
a_{n-1} y_{n-1}+b_{n} y_{n}+a_{n} y_{n+1}=\left(z+z^{-1}\right) y_{n}, \quad n=1,2, \ldots, \quad a_{0}=1,
$$

has two "distinguished" solutions. The first one, known as the sine-type solution, is

$$
y_{n}=s_{n}(z)=p_{n-1}\left(z+\frac{1}{z}\right), \quad s_{0}=0, s_{1}=1,
$$

$p_{k}$ are orthonormal polynomials with respect to the spectral measure $\sigma(J)$. A fundamental result by Szegó concerns an asymptotic behavior of orthonormal polynomials with respect to "nice" measures with supp $\sigma \subset[-2,2]$. It was extended substantially in [7], [10], and [11], where a finite (respectively, infinite) number of mass points outside $[-2,2]$ is allowed. In our notation the Szegő asymptotics states that for $J \in \mathcal{R}$

$$
Q(z)=\lim _{n \rightarrow \infty} z^{n} p_{n}\left(z+\frac{1}{z}\right)=\frac{B(z, J)}{\left(1-z^{2}\right) D(z, J)}
$$


uniformly on the compact subsets of the unit disk $\mathbb{D}$.

The second solution is the Jost solution $y_{n}=\varphi_{n}$, defined by a specific asymptotic behavior at infinity:

$$
\lim _{n \rightarrow \infty} z^{-n} \varphi_{n}(z, J)=1
$$

uniformly on the compact subsets of the unit disk $\mathbb{D}$. The Jost solution exists under certain additional assumptions (cf. [17], formulae (13.9.2)-(13.9.4)), which are met for $J \in \mathcal{R} . \varphi_{0}$ is called the Jost function. For an exhaustive treatment of the Szegó and the Jost asymptotics see [1] and Section 13.9 in [17].

The relation between the Szegó asymptotics and the Jost function is given by (see, e.g., [2] and Theorem 13.9.2 in [17])

$$
\varphi_{0}(z)=\left(1-z^{2}\right) Q(z)=\frac{B(z, J)}{D(z, J)}=(1-z)^{\gamma_{1}(J)}(1+z)^{\gamma_{2}(J)} \frac{B(z, J)}{D_{0}(z, J)} .
$$

We define the scattering function $s$ as in (16). By (19), we get

$$
\begin{aligned}
s(t, J) & =\frac{(1-t)^{\gamma_{1}(J)}(1+t)^{\gamma_{2}(J)}}{(1-\bar{t})^{\gamma_{1}(J)}(1+\bar{t})^{\gamma_{2}(J)}} \frac{D_{0}(\bar{t}, J)}{D_{0}(t, J)} B^{2}(t, J) \\
& =(-1)^{\gamma_{1}(J)} t^{\gamma_{1}(J)+\gamma_{2}(J)} \frac{D_{0}(\bar{t}, J)}{D_{0}(t, J)} B^{2}(t, J) .
\end{aligned}
$$

Clearly, $s(\bar{t})=\overline{s(t)}=s^{-1}(t)$.

Theorem 2.1. The scattering function s, defined as in (20), of a Jacobi matrix $J \in \mathcal{R}$, belongs to $B_{2}^{1 / 2}$ and admits a unique representation

$$
s(t, J)=(-1)^{\gamma_{1}(J)} t^{M} e^{-i v(t)}, \quad M=2 N+\gamma_{1}(J)+\gamma_{2}(J) \in \mathbb{Z}_{+},
$$

$v$ satisfies

$$
v(t)=\overline{v(t)}=-v(\bar{t}), \quad v \in B_{2}^{1 / 2} .
$$

Proof. By (10) and (11), we get that

$$
\frac{D_{0}(\bar{t}, J)}{D_{0}(t, J)}=\frac{\overline{D_{0}(t, J)}}{D_{0}(t, J)}=e^{-i v_{0}(t)} .
$$

Since $u_{0}=\log \hat{\rho}_{0} \in B_{2}^{1 / 2}$, and the Hilbert transform is bounded (isometric) in $B_{2}^{1 / 2}$, then $v_{0} \in B_{2}^{1 / 2}$. Since $u_{0}$ is symmetric, $v_{0}$ is antisymmetric.

Next,

$$
B^{2}(t, J)=t^{2 N}\left(\prod_{k=1}^{N} \frac{1-z_{k}(J) \bar{t}}{1-z_{k}(J) t}\right)^{2}=t^{2 N} e^{-i v_{1}(t)},
$$


and since $z_{k}(J) \in \mathbb{D}$, then $v_{1} \in B_{2}^{1 / 2}$ and it is antisymmetric. We put $v=v_{0}+v_{1} \in$ $B_{2}^{1 / 2}$. Since $v$ is a real function, we get (see, e.g., Proposition 6.1.11 in [16]) that $e^{-i v} \in B_{2}^{1 / 2}$. Therefore, $s \in B_{2}^{1 / 2}$

The uniqueness follows from a slight refinement of Peller's theorem (Corollary 7.8.2 in [12]), which states that an arbitrary function

$$
h \in B_{2}^{1 / 2}, \quad h(\bar{t})=\overline{h(t)}=h^{-1}(t)
$$

a.e. on $\mathbb{T}$, admits a unique representation

$$
h(t)=(-1)^{\gamma} t^{j} e^{-i w(t),}
$$

where $\gamma=0$ or $1, j \in \mathbb{Z}$ an integer number, $w$ satisfies (22). A pair $(\gamma, j)$ can be viewed as an index of $h$.

Let us turn to numbers $\mu_{k}(J)(15)$. In his version of the scattering theory for Jacobi matrices (2) Guseinov suggested the normalizing constants

$$
m_{k}(J)=\sum_{n=1}^{\infty}\left|\varphi_{n}\left(z_{k}(J), J\right)\right|^{2}, \quad k=1,2, \ldots, N,
$$

as a part of the scattering data. We show that these values agree with the numbers $\mu_{k}(J)$.

Proposition 2.2. Let $J \in \mathcal{R}$. Then $\mu_{k}(J)=m_{k}(J), k=1,2, \ldots, N$.

Proof. Let $s_{n}$ be defined as in (17). It is known from the general theory of Jacobi matrices and orthogonal polynomials, that the vectors

$$
\Pi_{k}=\left\{s_{n}\left(z_{k}(J)\right)\right\}_{n \geq 1}=\left\{p_{n}\left(\lambda_{k}\right)\right\}_{n \geq 0} \in \ell,
$$

so $\Pi_{k}$ are eigenvectors of $J$ with the corresponding eigenvalues $\lambda_{k}$. Furthermore,

$$
\frac{1}{\sigma_{k}(J)}=\sum_{n=1}^{\infty}\left|s_{n}\left(z_{k}(J)\right)\right|^{2}=\sum_{n=0}^{\infty}\left|p_{n}\left(\lambda_{k}\right)\right|^{2}
$$

On the other hand, $\varphi_{0}\left(z_{k}(J)\right)=0$, and so $\Phi_{k}=\left\{\varphi_{n}\left(z_{k}(J), J\right)\right\}_{n \geq 1}$ are also eigenvectors of $J$ for the same eigenvalues. Hence, $\Phi_{k}=c_{k} \Pi_{k}$, and we find the constants $c_{k}$ from the initial data $s_{1}=1$, so that $c_{k}=\varphi_{1}\left(z_{k}(J), J\right)$. By (24) and (25) $m_{k}(J)=\left|\varphi_{1}\left(z_{k}(J), J\right)\right|^{2} \sigma_{k}^{-1}(J)$.

It remains to express $\varphi_{1}$ in terms of the spectral data. Once the Jost asymptotics exists for $J \in \mathcal{R}$, the Jost solution $\varphi_{n}$ is proportional to the Weyl solution

$$
w_{n}(z)=\left(\left(z+z^{-1}-J\right)^{-1} e_{1}, e_{n}\right), \quad n=1,2, \ldots, \quad w_{0}=1,
$$


that is, $\varphi_{n}=\varphi_{0} w_{n}$. In particular,

$$
\varphi_{1}(z, J)=\varphi_{0}(z, J) w_{1}(z)=\varphi_{0}(z, J) M(z, J)
$$

where $M$ is the Weyl function for $J$,

$$
M(z, J)=\left(\left(z+z^{-1}-J\right)^{-1} e_{1}, e_{1}\right)=\int_{\mathbb{R}} \frac{\sigma(d \lambda)}{z+z^{-1}-\lambda}=\frac{\sigma_{k}(J)}{z+z^{-1}-\lambda_{k}}+\tilde{M}(z),
$$

$\tilde{M}$ is analytic at $z_{k}(J)$. So

$$
\varphi_{1}(z, J)=M(z, J) \frac{B(z, J)}{D(z, J)} .
$$

Since $\left.\lim _{z \rightarrow z_{k}}\left(z-z_{k}(J)\right) M(z, J)=\sigma_{k}(J)\left(1-z_{k}^{-2}(J)\right)\right)^{-1}$ we finally have

$$
\varphi_{1}\left(z_{k}(J), J\right)=\frac{\sigma_{k}(J)}{1-z_{k}^{-2}(J)} \frac{B^{\prime}\left(z_{k}(J), J\right)}{D\left(z_{k}(J), J\right)}
$$

as needed.

To complete the analysis of scattering data we prove the uniqueness theorem.

Theorem 2.3. Let $J_{l} \in \mathcal{R}, l=1,2$, have the same scattering data. Then $J_{1}=J_{2}$.

Proof. We want to make sure that $\sigma\left(J_{1}\right)=\sigma\left(J_{2}\right)$. It is clear from (20) that

$$
\frac{\overline{D_{0}\left(t, J_{1}\right)}}{D_{0}\left(t, J_{1}\right)}=\frac{\overline{D_{0}\left(t, J_{2}\right)}}{D_{0}\left(t, J_{2}\right)}
$$

By Proposition 1.5, $D_{0}^{ \pm 1} \in H^{2}$ In view of this, (26) implies that $D_{0}\left(J_{2}\right)=c D_{0}\left(J_{1}\right)$. Therefore, $D\left(J_{2}\right)=c D\left(J_{1}\right)$ for some $c>0$, and, hence, $\sigma_{a c}\left(J_{2}\right)=c^{2} \sigma_{a c}\left(J_{1}\right)$. Next, by (15) $\mu_{k}\left(J_{1}\right)=\mu_{k}\left(J_{2}\right)$ implies $\sigma_{k}\left(J_{2}\right)=c^{2} \sigma_{k}\left(J_{1}\right)$, and the normalizing condition

$$
\int_{-2}^{2} f\left(x, J_{1}\right) d x+\sum_{k=1}^{N} \sigma_{k}\left(J_{1}\right)=\int_{-2}^{2} f\left(x, J_{2}\right) d x+\sum_{k=1}^{N} \sigma_{k}\left(J_{2}\right)=1
$$

gives $c=1$, as needed.

\section{Inverse scattering}

Consider the following collection of data $\left\{\gamma_{1}, \gamma_{2} ; Z ; \mu_{1}, \ldots, \mu_{N} ; s\right\}$ :

(1) a pair of numbers $\left(\gamma_{1}, \gamma_{2}\right)$ from $\{0,1\} \times\{0,1\}$; 
(2) an arbitrary set of $N$ distinct points $Z=\left\{z_{k}\right\}_{k=1}^{N}$ in $(-1,1) \backslash\{0\}$;

(3) an arbitrary set of $N$ positive numbers $\mu_{k}$;

(4) a function $s \in B_{2}^{1 / 2},|s|=1$ a.e. on $\mathbb{T}$, with the index $\left(\gamma_{1}, 2 N+\gamma_{1}+\gamma_{2}\right)$, i.e.,

$$
s(t)=(-1)^{\gamma_{1}} t^{2 N+\gamma_{1}+\gamma_{2}} e^{-i \omega(t)}, \quad \omega(t)=\overline{\omega(t)}=-\omega(\bar{t}), \quad \omega \in B_{2}^{1 / 2} .
$$

Theorem 3.1. There exists a unique Jacobi matrix $J \in \mathcal{R}$, for which the above collection is the scattering data.

Proof. As in the proof of Theorem 2.1 (see (20) and (23)) we can write

$$
s(t)=\frac{(1-t)^{\gamma_{1}}(1+t)^{\gamma_{2}}}{(1-\bar{t})^{\gamma_{1}}(1+\bar{t})^{\gamma_{2}}} B^{2}(t, Z) e^{-i v_{0}(t)},
$$

$v_{0}$ is subject to (22). The Fourier series for $v_{0}$ is

$$
v_{0}(t)=\sum_{n \in \mathbb{Z}} v_{0, n} t^{n}, \quad v_{0,-n}=\overline{v_{0, n}}=-v_{0, n},
$$

so $v_{0,0}=0$. Take $u_{0}$ such that $v_{0}$ is its harmonic conjugate. Then

$$
u_{0}(\bar{t})=\overline{u_{0}(t)}=u_{0}(t)
$$

and

$$
u_{0}(t)=\sum_{n \in \mathbb{Z}} u_{0, n} t^{n}, \quad u_{0,-n}=\overline{u_{0, n}}=u_{0, n} .
$$

Note that $u_{0}$ is defined up to an additive real constant $u_{0,0}$, which will be chosen later on from the normalization condition.

Define a function $\rho_{0}$ on $[-2,2]$ by $\hat{\rho}_{0}=e^{u_{0}}$ and put

$$
\rho(x)=\frac{\rho_{0}(x)}{(2-x)^{\gamma_{1}}(2+x)^{\gamma_{2}}}, \quad f(x)=\frac{1}{2 \pi} \rho(x) \sqrt{4-x^{2}},
$$

both functions $\rho$ and $f$ are defined up to the factor $C=e^{u_{0,0}}$ Next, write

$$
\begin{aligned}
D_{0}(z) & =\exp \left\{\frac{1}{2} \int_{\mathbb{T}} \frac{t+z}{t-z} u_{0}(t) m(d t)\right\}=\exp \left\{\frac{u_{0}(z)+i v_{0}(z)}{2}\right\}, \\
D(z) & =\frac{D_{0}(z)}{(1-z)^{\gamma_{1}}(1+z)^{\gamma_{2}}},
\end{aligned}
$$

and put

$$
\sigma_{k}=\mu_{k}\left|\frac{D\left(z_{k}\right)}{B^{\prime}\left(z_{k}\right)}\right|^{2}\left|1-z_{k}^{-2}\right|^{-2}>0, \quad k=1,2, \ldots, N,
$$


the latter values are defined up to the same factor $C$, which is now taken from

$$
\int_{-2}^{2} f(x) d x+\sum_{k=1}^{N} \sigma_{k}=1 .
$$

Since $v_{0} \in B_{2}^{1 / 2}$, then so is $u_{0}$, and by Ryckman's theorem the measure $\sigma=$ $\left\{f,\left\{\sigma_{k}\right\}\right\}$ is the spectral measure of some Jacobi matrix $J \in \mathcal{R}$. By construction, $\left\{\gamma_{1}, \gamma_{2} ; Z ; \mu_{1}, \ldots, \mu_{N} ; s\right\}$ is the scattering data for $J$, and $J$ is unique by Theorem 2.3. The proof is complete.

\section{References}

[1] D. Damanik and B. Simon, Jost functions and Jost solutions for Jacobi matrices, I. A necessary and sufficient condition for Szegó asymptotics. Invent. Math. 165 (2006), 150. MR 2221136 Zbl 1122.47029

[2] D. Damanik and B. Simon, Jost functions and Jost solutions for Jacobi matrices, II. Decay and analyticity. Internat. Math. Res. Notices 2006 (2006) No.5, Art. ID 19396, 1-32. MR 2219207 Zbl 1122.47030

[3] I. Egorova, J. Michor, and G. Teschl, Scattering theory for Jacobi operators with quasi-periodic background. Comm. Math. Phys. 264 (2006), 811-842. MR 2217293 Zbl 1115.39025

[4] J. S. Geronimo, A relation between the coefficients in the recurrence formula and the spectral function for orthogonal polynomials. Trans. Am. Math. Soc. 260 (1980), 65-82. MR 0570779 Zbl 0445.42002

[5] J. S. Geronimo, Scattering theory, orthogonal polynomials, and $q$-series. SIAM J. Math. Anal. 25 (1994), 392-419. MR 1266566 Zbl 0802.42019

[6] J. S. Geronimo and P. Nevai, Necessary and sufficient conditions relating the coefficients in the recurrence formula to the spectral function for orthogonal polynomials. SIAM J. Math. Anal. 14 (1983), 622-637. MR 0697531 Zbl 0517.42031

[7] A. A. Gonchar, On convergence of Padé approximants for some classes of meromorphic functions. Sb. Math 97 (1975), 607-629. English transl.: Math. USSR Sb. 26 (1975), 555-575. MR 0387552 Zbl 0341.30029

[8] G. Sh. Guseinov, The determination of an infinite Jacobi matrix from the scattering data. Soviet. Math. Dokl. 17 (1976), 596-600. MR 0405160 Zbl 0336.47021

[9] G. Sh. Guseinov, The scattering problem for an infinite Jacobi matrix. Izv. Akad. Nauk Armyan. SSR Ser. Mat. 12 (1977), 365-379. In Russian. MR 0559594

[10] E. M. Nikishin, The discrete Sturm-Liouville operator and some problems of function theory. Trudy Sem. Petrovsk. 10 (1984), 3-77. English transl.: Soviet Math. 35 (1987), 2679-2744. MR 0778879

[11] F. Peherstorfer and P. Yuditskii, Asymptotics of orthogonal polynomials in the presence of a denumerable set of mass points. Proc. Am. Math. Soc. 129 (2001), 3213-3220. MR 1844996 Zbl 0976.42012 
[12] V. V. Peller, Hankel Operators and their applications. Springer Verlag, New York, 2003. MR 1949210 Zbl 1030.47002

[13] E. Ryckman, A strong Szegő theorem for Jacobi matrices. Comm. Math. Phys. 271 (2007), 791-820. MR 2291796 Zbl 1133.47025

[14] E. Ryckman, Erratum: A strong Szegő theorem for Jacobi matrices. Comm. Math. Phys., 275 (2007), 581-585. MR 2335786 Zbl 1137.47025

[15] E. Ryckman, Two spectral equivalences for Jacobi matrices. University of California Los Angeles, 2007. Ph.D. thesis. MR 2710782

[16] B. Simon, Orthogonal polynomials on the unit circle, Part 1: Classical Theory. Amer. Math. Soc., Providence (RI), 2005. MR 2105088 Zbl 1082.42020

[17] B. Simon, Orthogonal polynomials on the unit circle, Part 2: Spectral Theory. American Mathematical Society, Providence (RI), 2005. MR 2105089 Zbl 1082.42021

Received July 20, 2010; revised November 30, 2010

Leonid Golinskii, Mathematics Division, Institute for Low Temperature Physics and Engineering, 47 Lenin Avenue, Kharkov 61103, Ukraine

E-mail: leonid.golinskii@gmail.com

Alexander Kheifets, Department of Mathematics, University of Massachusetts Lowell, 01854, U.S.A.

E-mail: Alexander_Kheifets@uml.edu

Petro Yuditskiy, Institute for Analysis, Johannes Kepler University Linz, 4040 Linz, Austria

E-mail: petro.yuditskiy@jku.at 